\title{
Quenched disorder in a liquid-crystal biosensor: Adsorbed nanoparticles at confining walls
}

\author{
O. Guzmán, ${ }^{\text {a) }}$ N. L. Abbott, and J. J. de Pablob) \\ Department of Chemical and Biological Engineering, University of Wisconsin, Madison, \\ Wisconsin 53706-1691
}

(Received 3 November 2004; accepted 1 March 2005; published online 11 May 2005)

\begin{abstract}
We analyze the response of a nematic liquid-crystal film, confined between parallel walls, to the presence of nanoscopic particles adsorbed at the walls. This is done for a variety of patterns of adsorption (random and periodic) and operational conditions of the system that can be controlled in experimental liquid-crystal-based devices. We compute simulated optical textures and the total optical output of the sensor between crossed polars, as well as the correlation function for the liquid-crystal tensor order parameter; we use these observables to discuss the gradual destruction of the original uniform orientation. For large concentrations of particles adsorbed in random patterns, the liquid crystal at the center of the sensor adopts a multidomain state, characterized by a small correlation length of the tensor order parameter, and also by a loss of optical anisotropy under observation through crossed polars. In contrast, for particles adsorbed in periodic patterns, the nematic at the center of the cell can remain in a monodomain orientation state, provided the patterns in opposite walls are synchronized. (c) 2005 American Institute of Physics.
\end{abstract}

[DOI: $10.1063 / 1.1896354]$

\section{INTRODUCTION}

Sensors based on liquid crystals (LCs) represent a promising technology for the detection of particles of nanometerscale dimensions, including proteins and viruses. These sensors consist of a thin film of a nematic LC confined between two aligning surfaces; they rely on the long-range orientational order of the nematic to report binding of particles at specific receptors on the confining walls. ${ }^{1-3}$

In the absence of bound particles, the walls force the nematic to adopt a uniform orientation state, with a constant director field $\mathbf{n}(\mathbf{r})$. When observed through crossed polars, a system in this state exhibits a uniform optical texture. If the polars are rotated, one observes a sinusoidal modulation of the transmitted light.

Particles adsorbed at the walls of the sensor perturb the local orientation of the liquid crystal. The resulting distortions can extend over long distances, thereby disorganizing the uniform director field and producing characteristic signatures that can be read by optical means. As an instance, when the amount of particles bound at the surface is sufficiently large, the system adopts a multidomain conformation that manifests itself as a gradual loss of the anisotropy (modulation) of transmission of light between polars. ${ }^{2}$

From a practical point of view, the ability to predict and control the onset of the destruction of the uniform nematic state could permit design of sensing devices with improved sensitivity. In addition, from a fundamental perspective, it is of interest to investigate the effects of quenched disorder on the stability of the uniform state of the order parameter. ${ }^{4-7}$

In the pioneering work of Imry and $\mathrm{Ma}^{4}{ }^{4}$ scaling argu-

\footnotetext{
a)Electronic mail: orlando@che.wisc.edu

${ }^{b)}$ Electronic mail: depablo@engr.wisc.edu
}

ments were used to prove the instability of the uniform, monodomain state against arbitrarily weak random fields that span a system. This result holds for domains of sufficiently large size $L$ in systems with dimensionality $d<4$.

More recently, the instability of the monodomain bulk state against surface randomness has also been established. ${ }^{6}$ The scaling argument can be summarized as follows: Consider a region of size $L$ near the surface and compare the energies of the uniform and multidomain states. For an order parameter with continuous symmetry (such as $\mathbf{n}$ or $\mathbf{Q}$ ), the long-range elastic-energy contribution scales as $E_{\text {elast }} \sim L^{d-2}$, while the energy due to interaction with the surface scales as $E_{\text {surf }} \sim L^{(d-1) / 2}$. The ratio $E_{\text {surf }} / E_{\text {elast }}$ scales as $L^{3 / d-1}$. Hence, for $d<3$, the surface-energy penalty is larger than the elastic-energy cost of setting up domains of sufficiently large size $L$, rendering the uniform state unstable. The borderline case $d=3$ turns out to behave in the same way, rendering the uniform state unstable against arbitrarily weak perturbations for all $d \leqslant 3 .^{6}$ However, the previous analysis assumes that domains of an unspecified (but large) size can be produced; it may not hold for situations in which there is an upper limit to the size of domains, as is the case in a confined system.

In a previous publication, ${ }^{8}$ we have presented the results of a coarse-grained simulation of a confined LC-based sensor. We analyzed the time evolution of the average domain size $L_{D}$ as a function of the surface coverage $c$ of nanoparticles adsorbed at the confining walls. A critical surface coverage $c_{c}$ was identified, above which the nematic ceases to exhibit long-range uniformity. The optical textures of the simulated sensor were similar to those observed in experiments, but quantitative measures of the optical output of the sensor were not discussed.

In this work, we return to that model to identify some of 
the factors that can facilitate the destruction of the monodomain state and enhance the sensitivity of the sensor. Specifically, we examine the effects of altering variables that can be controlled during the design and operation of an experimental liquid-crystal-based sensor:

(1) the scalar order parameter $S$ of the nematic,

(2) the separation $Y$ between confining walls,

(3) the number $m$ of contiguous particles adsorbed as a cluster, and

(4) the lag $\delta_{x}$ between periodic-adsorption patterns at opposite walls.

The response of the sensor is analyzed in terms of the simulated optical textures of the system between crossed polars, the corresponding intensities of the transmitted light, as well as the correlation function of the tensor order parameter, from which the average domain size $L_{D}$ can be found.

While this two-dimensional (2D) model has limitations that do not permit a complete comparison with experiments in three dimensions (3Ds), it also has proven useful in several regards: first, it permits a fast exploration of the large parameter space where a LC biosensor might operate; second, it provides insights that will allow more demanding 3D modeling efforts to concentrate in the most interesting regions of the parameter space; and, finally, it has been useful in interpreting recent experimental work. ${ }^{9}$ Hence, we leave for future publications detailed comparisons of our current calculations with three-dimensional models and the corresponding experiments.

The paper is organized as follows: Sec. II describes the model LC sensor, the coarse-grained theory governing its time evolution, and the parameters used to simulate each system. In Sec. III we present results for the response of the sensor to variations of the specified operational variables $\left(S, Y, m, \delta_{x}\right)$, and discuss their interpretation in terms of the gradual destruction of the uniform-oriented texture. Finally, Sec. IV presents our conclusions and suggests some possible extrapolations for the three-dimensional case.

\section{METHODS}

\section{A. The model sensor}

The model sensor considered in this work mimics the geometry of LC-based devices discussed in recent literature. ${ }^{1-3}$ The experimental setup consists of a thin film of mesogenic fluid (e.g., pentylcyanobiphenyl, 5CB) confined between two parallel walls, which are separated by a distance of the order of tens of microns. The walls consist of gold surfaces coated by self-assembled monolayers (SAMs) of organosulfur compounds (e.g., alkanethiols); their topography and chemistry can be controlled to ensure planar anchoring of the liquid crystal. ${ }^{3}$

In the absence of bound particles, the planar anchoring at the walls induces a uniform planar texture that extends throughout the device. When proteins or viruses bind to specific receptors at the surface, they perturb the local anchoring conditions; this perturbation is transmitted by the nematic to regions far away from the substrate, giving rise to an amplification mechanism that facilitates optical detection: while

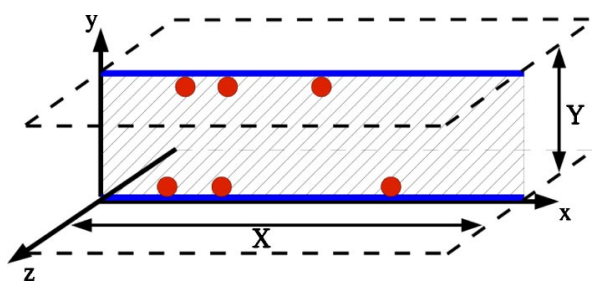

FIG. 1. Schematic view of a model LC sensor that is invariant along the $z$ axis. Two planar walls confine the LC in the $y$ direction, while periodic boundary conditions are applied in the $x$ direction. Particles of radius $R$ can be adsorbed at the confining walls.

typical targets, such as proteins, have sizes on the order of tens of nanometers, the scale of the optical signatures observed in the sensor is of the order of tenths of a millimeter.

If we were to numerically simulate the dynamics of the system, and represent atomically the adsorbed particles and the liquid crystal, the computational demands would be formidable. Coarse-grained methods have been developed to surpass this limitation; among others, we can mention lattice models $^{10}$ and continuum alignment tensor-field theories. ${ }^{11,12}$ In previous studies we have shown that results from molecular simulations can be combined with coarse-grained theories, and have found that the latter provide an accurate representation of the structure of defects and director textures down to length scales of a few molecular diameters. ${ }^{13-15} \mathrm{We}$ therefore resort to a coarse-grained approach in which we track the state of the liquid crystal through its tensor orderparameter field, ${ }^{16,17}$ and the adsorbed particle is treated as a solid object.

Bridging the difference of the scales between the size of the adsorbed particle and the scale of the optical textures requires a resolution of the order of $10^{-3}$ of the sensor cell size. In three dimensions, this is computationally demanding and is beyond our computational resources. As an alternative, we study the dynamics of a quasi-2D system. The use of this type of quasi-2D systems has proved very useful in the past for the study of phase ordering kinetics of nematic fluids, ${ }^{18,19}$ the analysis of defect structures and forces that arise in the presence of colloidal particles, ${ }^{20,21}$ as well as the investigation of backflow. ${ }^{22}$ For these quasi-2D systems (see Fig. 1), one considers a rectangular area in the $x y$ plane, representing a section through a three-dimensional system that is invariant along the $z$ axis.

In experiments, bound particles are immobilized at the confining walls prior to the filling of the sensor with a liquid crystal. In this sense, the disorder introduced at the walls is quenched: the state of the system depends on random variables (the positions of the bound particles) that are drawn from a particular distribution, but those variables remain fixed for any physical realization of the sensor. ${ }^{23,24}$ Therefore, in each of our simulations, we create an initial configuration of adsorbed particles, and keep it fixed as the liquid crystal evolves. The physical properties of the system are computed as averages over both particle configurations and initial conditions of the nematic.

Besides individual random adsorption, we consider also random adsorption of clusters of particles, since it is common for biological particles (the targets of the sensors) to 
aggregate and adsorb in groups. We also consider adsorption in periodic patterns because, in experiments, it is possible to organize the receptors on the confining surface in a periodic configuration, and therefore indirectly create a periodic array of adsorbed particles.

\section{B. The evolution equation}

We compute the evolution of the liquid crystal with a dynamic theory for its tensor order-parameter field $\mathbf{Q}(\mathbf{r}, t)$. The local values of the scalar order parameter $S$ and the director $\mathbf{n}$ can be obtained from $\mathbf{Q}$ through its largest eigenvalue $2 S / 3$ and its associated eigenvector, respectively. ${ }^{16}$ The theory corresponds to a particular case of the Beris-Edwards formulation of the thermodynamics of fluids with internal microstructure. ${ }^{25}$ In this formulation, the functional derivative of the free energy $\mathcal{F}$ with respect to the order parameter drives the evolution of $\mathbf{Q}$ :

$$
\frac{\partial \mathbf{Q}}{\partial t}=\Gamma\left[-\frac{\delta \mathcal{F}}{\delta \mathbf{Q}}+\frac{1}{3} \operatorname{Tr}\left(\frac{\delta \mathcal{F}}{\delta \mathbf{Q}}\right) \mathbf{I}\right]
$$

The coefficient $\Gamma$ is given by $\Gamma=6 D^{*} /\left[1-3 \operatorname{Tr}\left(\mathbf{Q}^{2}\right)\right]^{2}$, where $D^{*}$ is the rotational diffusion for the nematic. In these equations, it is assumed that $\delta \mathcal{F} / \delta \mathbf{Q}$ has been symmetrized.

This work only considers the case of strong anchoring at all surfaces; hence, surface free-energy contributions need not be included, provided that the initial configurations satisfy the anchoring at every confining surface. Then the free energy $\mathcal{F}$ of the liquid crystal includes two contributions. One corresponds to the short-range bulk interactions responsible for the $I-N$ transition; the other one arises from the elasticity of the liquid crystal:

$$
\mathcal{F}=\int_{V} F_{s}+F_{e} d \mathbf{r}
$$

A Landau-de Gennes expansion is used to represent the short-range free-energy density:

$$
\begin{aligned}
F_{s}= & \int \frac{A}{2}\left(1-\frac{U}{3}\right) \operatorname{Tr}\left(\mathbf{Q}^{2}\right)-\frac{A U}{3} \operatorname{Tr}\left(\mathbf{Q}^{3}\right) \\
& +\frac{A U}{4} \operatorname{Tr}\left(\mathbf{Q}^{2}\right)^{2} d \mathbf{r} .
\end{aligned}
$$

The phenomenological coefficients $A$ and $U$ depend on the liquid crystal of interest; $A$ controls the energy scale of the model, while $U$ controls the value of the scalar order parameter in the bulk: ${ }^{25}$

$$
S_{\text {bulk }}(U)=\frac{1}{4}(1+3 \sqrt{1-8 / 3 U}) .
$$

In this model, the system is isotropic for $0<U<U_{I N}=2.7$, and nematic for $U>U_{I N}$.

The free-energy penalty for gradients in the tensor orderparameter field results in an elastic contribution of the form ${ }^{26}$

$$
F_{e}=\int \frac{L_{1}}{2} Q_{i j, k} Q_{i j, k}+\frac{L_{2}}{2} Q_{i k, i} Q_{j k, j}+\frac{L_{3}}{2} Q_{i j} Q_{k l, i} Q_{k l, j} d \mathbf{r},
$$

where $Q_{i j, k}=\partial_{k} Q_{i j}$. The coefficients $L_{i}$ are related to the splay $\left(K_{1}\right)$, twist $\left(K_{2}\right)$, and bend $\left(K_{3}\right)$ elastic constants:

$$
\begin{aligned}
& L_{1}=\frac{3 K_{2}-K_{1}+K 3}{6 S^{2}}, \\
& L_{2}=\frac{K_{1}-K_{2}}{S^{2}}, \\
& L_{3}=\frac{K_{3}-K_{1}}{2 S^{3}} .
\end{aligned}
$$

In this work, we use the one-elastic-constant approximation, ${ }^{16,27}$ where the splay, twist, and bend elastic constants have a common value $K$ that depends quadratically on the scalar order parameter, ${ }^{28}$

$$
K=2 S^{2} L_{1},
$$

and the elastic free-energy density reduces to

$$
F_{e}=\int \frac{L_{1}}{2} Q_{i j, k} Q_{i j, k} d \mathbf{r} .
$$

We do not expect that by introducing more than one elastic constant the general behavior of the biosensor model will change. For instance, theoretical studies for randomly confined nematics indicate that taking different values for the elastic constants $\left(K_{1}, K_{3}>K_{2}\right)$ does not change the universality class of the model. ${ }^{29}$ However, for future, detailed comparisons between three-dimensional models and experimental results we do expect that the general form given in Eq. (5) will be required.

When the functional derivatives in Eq. (1) are evaluated, one obtains a partial differential equation for $\mathbf{Q}$,

$$
\begin{aligned}
\frac{\partial \mathbf{Q}}{\partial t}= & -\Gamma\left\{A(1-U / 3) \mathbf{Q}-A U\left[\mathbf{Q}^{2}-\operatorname{Tr}\left(\mathbf{Q}^{2}\right)(\mathbf{Q}+\mathbf{I} / 3)\right]\right. \\
& \left.-L_{1} \nabla^{2} \mathbf{Q}\right\},
\end{aligned}
$$

which can be solved numerically. In this work we use a finite-difference, explicit Euler method that has been described in previous publications. ${ }^{8,13}$ For brevity, in Sec. II C, we just specify the conditions for which the calculations were performed.

\section{Parameters for the simulations}

In Ref. 8 a set of parameters was chosen to represent a low molecular weight liquid crystal at a high value $(S$ $=0.81$ ) of the scalar order parameter: $A=1, L_{1}=0.55, D^{*}$ $=0.35$, and $U=6$. In this work we adopt the same values for $A, L_{1}$, and $D^{*}$, but for $U$ we use two different values: $U$ $=4.8$ and $U=3.1$. The value $U=4.8$ yields a bulk scalar order parameter of $S=0.75$ and was adopted because, for this value of $S$, we have recently completed a multiscale analysis of defect structures around colloidal particles. ${ }^{15}$ Hence, this choice of $U$ will facilitate future comparisons to results of molecular simulations. Another set of simulations was run at 
$U=3.1$ in order to observe the effect of approaching the isotropic-nematic transition, since the limit of metastability of the isotropic phase is $U^{*}=3.0$.

From these phenomenological parameters, it is possible to extract characteristic time and length scales and to map them into those of a typical liquid crystal. For instance, we can define a characteristic length for the changes in the tensor order parameter as ${ }^{30}$

$$
\xi=\sqrt{18 L_{1} / A U}
$$

and a characteristic time scale as

$$
\tau=\xi^{2} / 6 D^{*} L_{1} .
$$

The values of $\xi$ and $\tau$ at the $I-N$ transition are used in this paper as units for length and time: $\xi_{I N}=1$ and $\tau_{I N}=1$. Identifying these values with those of a typical nematic, ${ }^{16}\left(\xi_{I N}\right.$ $\approx 20 \mathrm{~nm}, \tau_{I N} \approx 10^{-2} \mathrm{~s}$ ), it is possible to translate the results of the theory into SI units and compare with experiments.

Computational resources limit the size and resolution of the systems that we can simulate. For this reason, we have chosen a system size that is about one-tenth of the size of an experimental device, but which we simulate using a sufficiently high resolution to avoid the artificial pinning of defects. Using the previous convention of units, the length of our periodic cell is $X=194.3 \approx 3890 \mathrm{~nm}$, while the wall separation takes one of two values, $Y=32.4 \approx 650 \mathrm{~nm}$ or $Y$ $=25.0 \approx 500 \mathrm{~nm}$. (The larger system corresponds to that studied in Ref. 8.) For the larger separation we use a grid of $1024 \times 171$ sites; for the smaller one the grid consists of $1024 \times 131$ sites. The resolution of the grid is therefore $\Delta r$ $=0.19 \approx 3.8 \mathrm{~nm}$. With these choices, in the absence of adsorbed particles, all defects are annihilated at long times and the system is observed to relax to a state of uniform texture. ${ }^{8}$

As discussed in Sec. II A, adsorbed nanoparticles are represented by fixing $N$ particles of radius $R=1.61$ $\approx 32.2 \mathrm{~nm}$ to each wall. The pattern of adsorption can be random or periodic. We define the surface coverage $c$ as

$$
c=2 R N / X \text {. }
$$

We sometimes refer to the surface coverage as the concentration of adsorbed particles.

In the simulation, once the position of the particles is fixed, initial configurations for the order-parameter field $\mathbf{Q}(\mathbf{r})$ are generated as follows: first, the tensor order parameter at the walls' and particles' boundaries is taken as

$$
\mathbf{Q}=S_{\text {bulk }}\left(\mathbf{n}_{0} \mathbf{n}_{0}-\mathbf{I} / 3\right),
$$

where $\mathbf{n}_{0}$ is the preferred orientation of strong anchoring and $S_{\text {bulk }}$ is given by Eq. (4). At the particles' surface, the anchoring is homeotropic and the preferred orientation $\mathbf{n}_{0}$ is a radial unit vector. This choice is motivated by recent experimental findings for the anchoring induced by vesicular stomatitis viruses. ${ }^{31}$ At the walls, the anchoring is planar and $\mathbf{n}_{0}$ is the unit vector along the $x$ axis.

For each of the remaining sites, we initialize $\mathbf{Q}$ to the form given in Eq. (15), but with $\mathbf{n}_{0}$ taken to be a different random orientation for each site. The system is then allowed to evolve with $U=2$ for a time $t_{\text {iso }}=20$, such that during the interval $-t_{\text {iso }} \leqslant t<0$ the liquid is isotropic. At time $t=0$, the system is quenched instantaneously to the nematic phase by setting $U=4.8$ (or $U=3.1$ ); the evolution of the liquid crystal is then analyzed from that starting point. We present only results for late times, for which the free energy, the optical texture, and the correlation length have reached a steady state. Typically, we have to wait a time on the order of $10 \tau_{I N}$ to ensure that we have reached this stage.

\section{RESULTS}

As described in the Introduction, we study the response of various systems to different concentrations of adsorbed particles. We start by arbitrarily selecting a system, called the reference system, against which all of the other systems are compared. For the reference system, we set the wall separation to $Y=32.4$, the scalar order parameter to $S=0.75$ (with $U=4.8$ ), and we specify an individual random adsorption at the walls. We then examine, one by one, a collection of systems that deviate from the reference in the following respect:

(1) scalar order parameter, $S=0.53$ (with $U=3.1$ ),

(2) wall separation, $Y=0.25$,

(3) adsorption in clusters of three particles, $m=3$,

(4) in-phase periodic patterns in opposite walls, and

(5) out-of-phase periodic patterns in opposite walls.

For the systems listed above, we have performed a series of simulations with surface coverages between $c=0.10$ and $c$ $=0.40$, in increments of 0.05 . (This is similar to the procedure used in Ref. 8.)

In the rest of this section we describe the response of the sensor to the variations listed above by visualizing the optical texture of the sensor under crossed polars, computing the correlation function for the tensor order parameter, and measuring the transversal- and lateral-optical outputs at each concentration.

\section{A. Lateral visualization}

The qualitative behavior of the system can be illustrated with visualizations of the optical texture of the liquid crystal. A lateral view of the system is obtained by cutting a thin slice (parallel to the $x y$ plane) and simulating the passage of light through this slice between crossed polars.

These visualizations are based on a tracing procedure, introduced by Nicholson, ${ }^{32}$ which has been previously applied to obtain simulated polarized light images of nematic liquid-crystal droplets. ${ }^{33}$ In this method, each cell is considered as a thin nematic plate of thickness $d$. Because the system is effectively $2 \mathrm{D}$, the local director $\mathbf{n}(x, y)$ is found to lie on the $x y$ plane. The angle between the polarizer's axis and $\mathbf{n}$ is denoted by $\alpha$; then the transmitted intensity is given by

$$
I=I_{0} \sin ^{2}(2 \alpha) \sin ^{2}(\epsilon / 2) .
$$

In the previous equation, $I_{0}$ is the intensity of the incident light, and $\epsilon$ is the phase shift introduced by the birefringence of the nematic.

The phase shift depends on the thickness of the slice, the wavelength, the angle between the director and the propagation direction, and the refractive indices of the material. Let 
$n_{\text {eff }}$ represent the effective extraordinary index that corresponds to propagation at an angle $\gamma$ with respect to the director $\mathbf{n}$,

$$
n_{\mathrm{eff}}(\gamma)=\left(\frac{\sin ^{2} \gamma}{n_{e}^{2}}+\frac{\cos ^{2} \gamma}{n_{o}^{2}}\right)^{-1 / 2}
$$

In this equation, $n_{e}$ is the extraordinary refractive index, and $n_{o}$ is the ordinary refractive index. Both are functions of the wavelength $\lambda$. Then the phase shift is given by

$$
\epsilon=\frac{2 \pi d}{\lambda}\left[n_{\mathrm{eff}}(\gamma)-n_{o}\right] .
$$

Since we are considering propagation with $\gamma=\pi / 2$ and crossed polars, the phase shift is constant and the transmitted intensity depends only on the local value of $\alpha$,

$$
I=I_{0} \sin ^{2}(2 \alpha) \sin ^{2}(\epsilon / 2) .
$$

Hence, regions where the director is vertical or horizontal will be dark in the visualizations, while regions with "diagonal" directors will be bright.

The optical textures obtained for the random-adsorption systems are very similar; we illustrate the general behavior in Fig. 2 for two cases: (a) the reference system and (b) the system with adsorption in clusters of three. ${ }^{34}$ In that figure, each texture corresponds to a different value of the surface coverage $c$, and one can see how the uniform orientation texture becomes disorganized as $c$ is increased. At low particle concentrations, the texture is mostly dark, except for a few bright regions. At larger concentrations, the bright regions grow in size and, in most cases, connect both sides of the system. This alternation of extended bright and dark zones signals the presence of multiple domains for the orientation of the director field.

The optical textures for periodic-adsorption patterns are considerably different from those corresponding to random adsorption. Figure 3 shows textures for systems with (a) zero lag between patterns in opposite walls, and (b) a lag equal to half of the distance between adjacent particles, $\delta_{x}=X / 2 N$. For the zero-lag system, we observe that the uniformity of the texture is preserved, even at a concentration $c=0.20$, where random-adsorption systems already exhibit nonuniform behavior. Even at $c=0.40$, the bright regions are confined to the proximity of the spherical particles, and they do not connect opposite walls. The out-of-phase system also remains organized at $c=0.20$, but, at the highest concentrations $(c=0.35,0.40)$ it has become disorganized, with bright domains spanning the gap between walls. It is important to state that, due to the incommensurability of the periods of the patterns and the cell size, these systems are only approximately periodic: due to periodic boundary conditions, the particles at the edges of the periodic cell have neighbors at distances slightly different from the rest. This is why the textures are richer at the edge of the periodic cell.

\section{B. Correlation functions and domain size}

In order to quantify the qualitative observations made in Sec. III A, we turn to the correlation function for the tensor order parameter, ${ }^{18}$
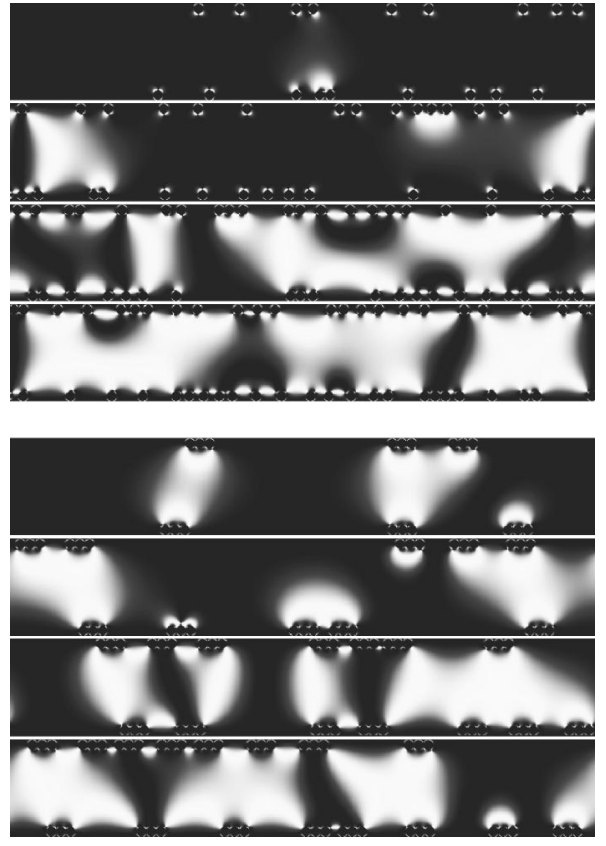

FIG. 2. Simulated lateral views (between crossed polars) for (a) the reference and (b) the system with adsorption in clusters of $m=3$ particles. For each panel, the surface coverage increases from top to bottom: $c=0.15,0.25$, 0.35 , and 0.40

$$
C(x, y)=\frac{\langle\mathbf{Q}(x, y): \mathbf{Q}(0, y)\rangle}{\langle\mathbf{Q}(0, y): \mathbf{Q}(0, y)\rangle} .
$$

The brackets denote an average over different configurations of the adsorbed particles and the initial field $\mathbf{Q}(x, y)$. In this work, the average is computed over five initial conditions with different particle and liquid-crystal configurations. We compute $C(x, y)$ only for those $y$ levels that do not intercept any particle.

As in our previous study, ${ }^{8}$ in order to investigate the response at the center of the cell, we compute the average of $C(x, y)$ over a narrow band of rows,
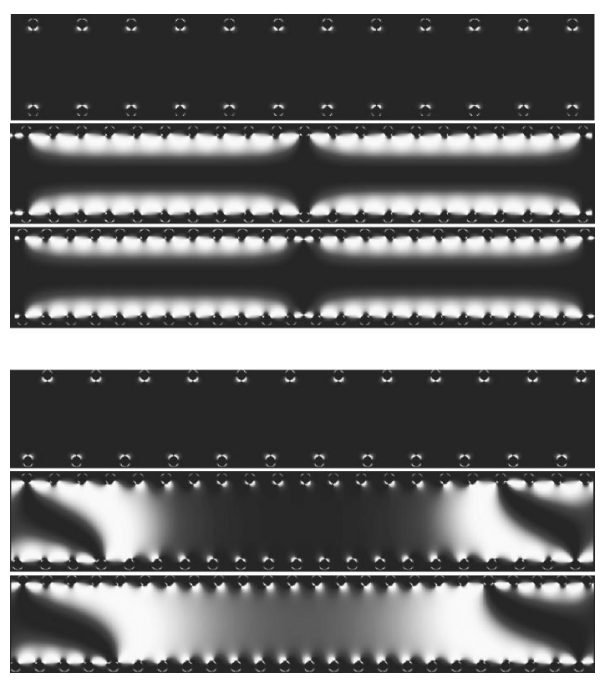

FIG. 3. Simulated lateral views (between crossed polars) for systems with periodic patterns of adsorption in opposite walls shifted by an amount (a) $\delta_{x}=0$ and (b) $\delta_{x}=X / 2 N$. For each panel, the surface coverage increases from top to bottom: $c=0.20,0.25,0.35$, and 0.40 . 

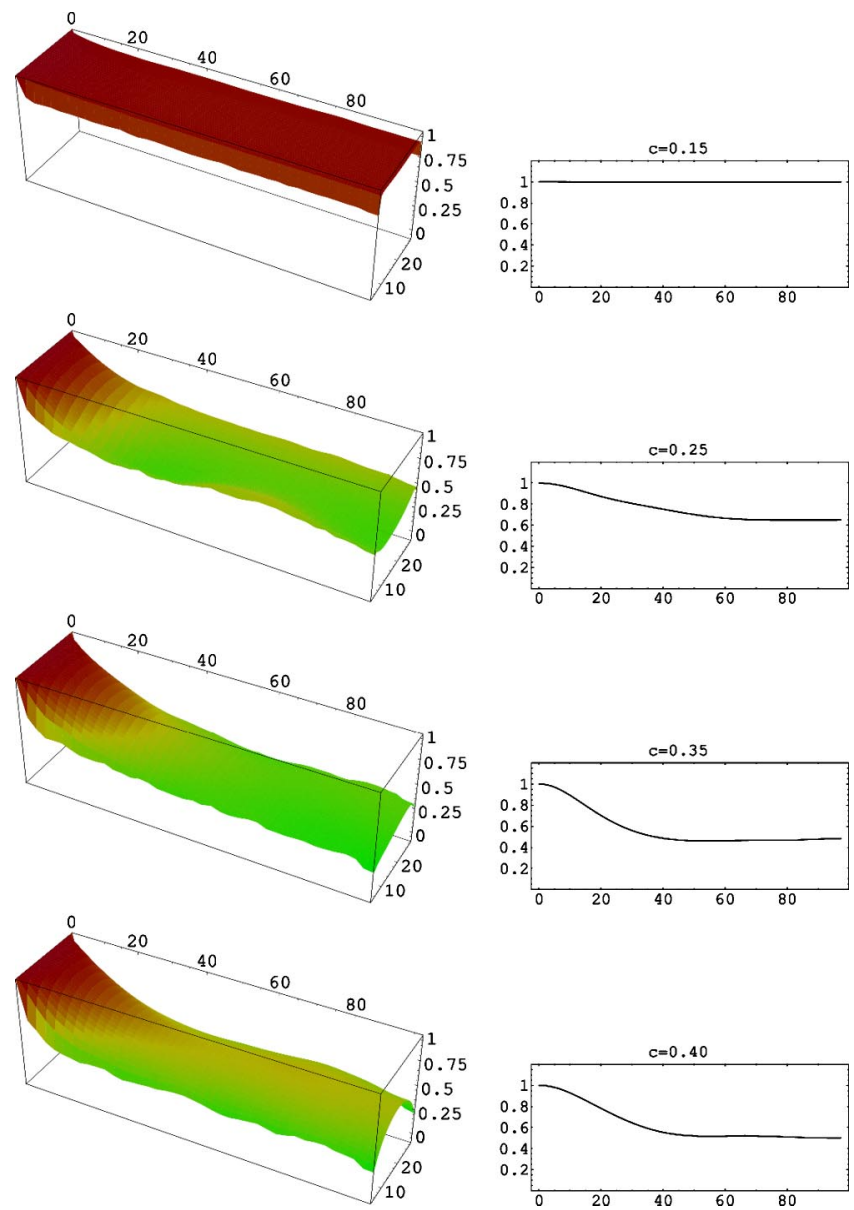

FIG. 4. Correlation functions for the reference system: the surfaces on the left correspond to $C(x, y)$, while the curve on the right corresponds to the central average $C(x)$. The surface coverage increases from top to bottom in each panel: $c=0.15,0.25,0.35$, and 0.40 .

$$
C(x)=\frac{1}{a} \int_{(Y-a) / 2}^{(Y+a) / 2} C(x, y) d y .
$$

In this work, the average is performed on the central $10 \%$ of the sensor; hence $a=L_{y} / 10$. A conventional definition of the average domain size $L_{D}$ has been given by Zapotocky et $a l .,{ }^{18}$

$$
C(x)_{\mid x=L_{D}}=1 / 2 .
$$

In other words, the domain size is the distance at which the correlation has decayed to one-half of its original value. If no solution can be found, we assume that the domains are larger than what can be observed, given the finite size of the periodic cell.

As one would expect from the optical textures, $C(x, y)$ behaves differently for the random-adsorption and periodicadsorption systems. Figure 4 (left) illustrates the typical behavior for the first group with the reference system: at low concentrations, there is little variation of $C(x, y)$ in either the $x$ or $y$ directions. As the concentration is increased, the variation along the $y$ axis remains relatively minor, but along the $x$ axis there is a noticeable decay towards a plateau value. This is shown in Fig. 4 (right) with plots of $C(x)$ at the same concentrations shown in Fig. 4 (left).
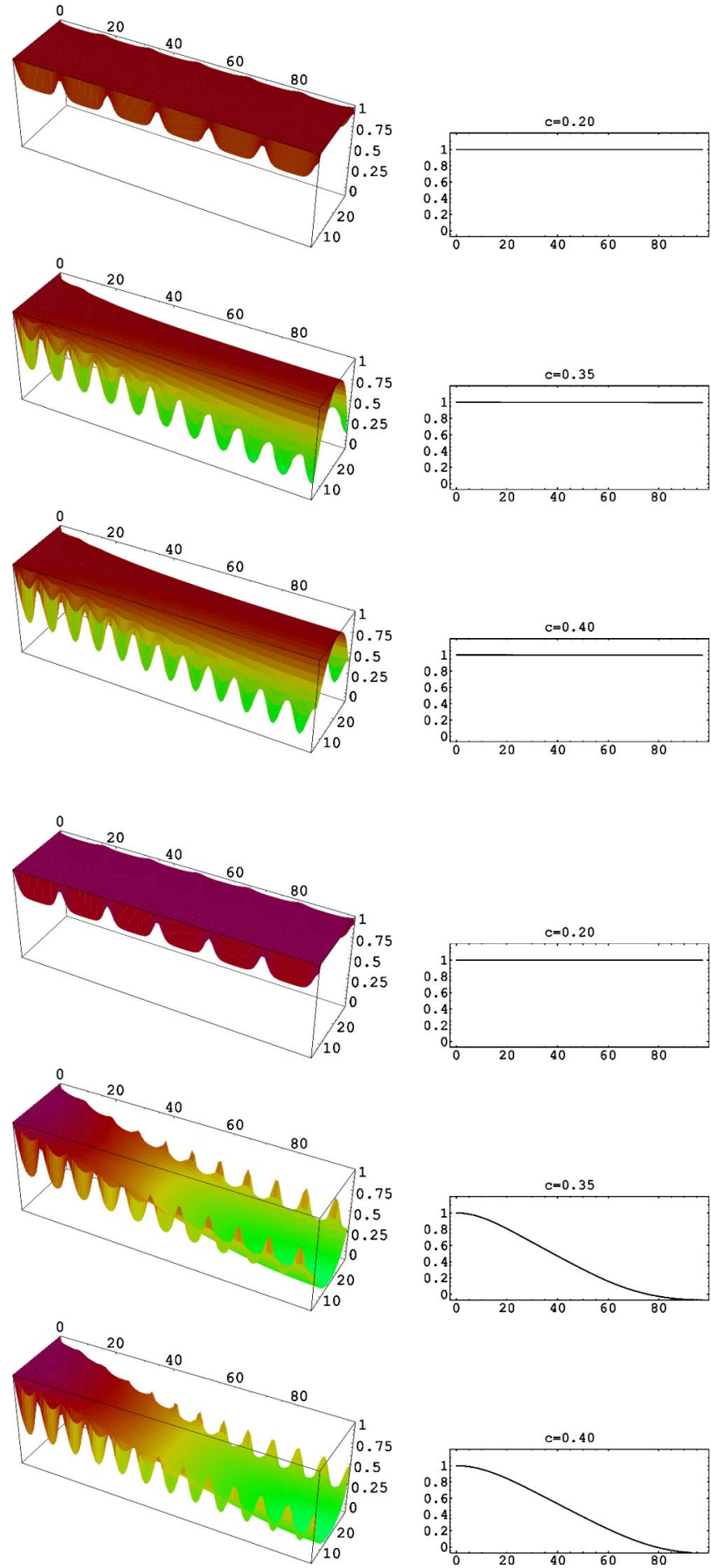

FIG. 5. Correlation functions for periodic systems with a phase shift of $\delta_{x}$ $=0$ (upper half) and $\delta_{x}=X / 2 N$ (lower half). For each system, the surface on the left corresponds to $C(x, y)$, while the curves on the right correspond to the central average $C(x)$. The surface coverage increases from top to bottom for each panel: $c=0.20,0.35$, and 0.40 .

The correlation functions of the periodic systems behave differently from those of the random systems; the precise behavior depends on the lag $\delta_{x}$. Figure 5 shows the correlation function $C(x, y)$ and the central average $C(x)$ for both periodic systems. A noticeable feature is the presence of periodic peaks in the correlation function $C(x, y)$, far away from the central rows, close to the surface of the particles. Their total number corresponds to the number of particles 
TABLE I. Average domain size $L_{D}$, as a function of the surface coverage $c$, for each of the simulated systems. A star indicates that $L_{D}$ is undefined since $C(x)$ does not decay below $1 / 2$.

\begin{tabular}{ccccccc}
\hline \hline$c$ & Reference & $S=0.53$ & $Y=25.0$ & $m=3$ & $\delta_{x}=0$ & $\delta_{x}=X / 2 N$ \\
\hline 0.00 & $*$ & $*$ & & & & \\
0.15 & $*$ & $*$ & $*$ & $*$ & & $*$ \\
0.20 & $*$ & $*$ & $*$ & $*$ & $*$ & $*$ \\
0.25 & 26.7 & $*$ & $*$ & $*$ & $*$ \\
0.30 & 30.0 & 38.8 & 25.1 & $*$ & $*$ & 38.2 \\
0.35 & 40.3 & $* 7.2$ & 27.7 & 22.8 & $*$ & 42.0 \\
0.40 & $*$ & $*$ & 29.6 & 29.6 & & \\
\hline \hline
\end{tabular}

adsorbed at each wall. [Since $C(x, y)$ is an even function of $x$, Fig. 5 only shows half of the domain and half of the peaks.]

For the in-phase system, $C(x, y)$ in the central rows remains very close to unity at concentrations where the random system exhibits a noticeable decay. This is consistent with the textures shown in Fig. 3. For the out-of-phase system, we observe that not only the peaks decay with $x: C(x, y)$ in the central region decays monotonically and reaches zero.

The average domain size $L_{D}$ is reported in Table I for all the simulated systems. This table shows that, at low values of $c, L_{D}$ is undefined since the correlation function does not decay below $1 / 2$. This can be interpreted as the preservation of the uniform orientation at the center of the cell. At larger values of the surface coverage, the function $C(x)$ does decay below the 0.5 level. We call the value of $c$ at which this change occurs the critical concentration, $c_{c}$. We can estimate $c_{c}$ as the average of the nearest two simulated concentrations. Using this measure, we can say that the critical concentration increases by $22 \%$ when the scalar order parameter is brought closer to the isotropic-nematic coexistence, from the reference value $S=0.75$ to $S=0.53$. The case of adsorption in clusters of $m=3$ particles is also interesting. From Table I, we can estimate that the critical coverage $\left.c_{c}\right|_{m=3}=0.325$ is larger by $44 \%$ than the corresponding value $\left.c_{c}\right|_{m=1}=0.225$ for the individual adsorption case. Indeed, using the data in Table I, we can see that the reference system has the smallest critical concentration among the simulated systems.

The definition of $c_{c}$ depends heavily on the requirement that the correlation $C(x)$ decays to values below $1 / 2$ before a solution for $L_{D}$ can be found. Hence, we would like to provide an alternative measure for the characteristic length of $C(x)$, one that is defined even when this correlation function does not decay below $1 / 2$. To this end, we modify Eq. (22) by taking into account that the "final" plateau value may not be zero:

$$
C(x)_{\mid x=L_{C}}=\frac{1+C(X / 2)}{2} .
$$

Table II presents the value of $C(X / 2)$. For the randomadsorption systems, the plateau value $C(X / 2)$ goes through a minimum and then increases again. As discussed in Ref. 8, this indicates that, as the surface becomes completely covered by particles, a uniform texture is slowly restored; this time, however, its orientation is governed by the anchoring properties of the particles and not by those of the surface.

The corresponding values of $L_{C}$ are presented in Table III. From the data in this table, we observe that even in those cases where $L_{D}$ is undefined, we can observe a progressive increment on $L_{C}$ as the number of adsorbed particles is increased. There are still instances where $L_{C}$ is undefined, but those are confined to cases where $C(x)$ does not decay at all, and remains constant and equal to unity.

As in the case of $C(X / 2)$, we observe that $L_{C}$ exhibits a minimum for the random-adsorption systems. In general, we can say that the effect of lowering $S$ is to increase the size of the domains; this is expected since at lower $S$ the coherence length $\xi$ is larger. Reducing the separation between walls has the opposite effect, that is, $L_{C}$ is reduced. For the periodic systems, $L_{C}$ is always undefined in the in-phase case, while it increases monotonically with $c$ for the out-of-phase system. These results suggest that it should be possible to obtain information about the morphology of the aggregates from the state of the liquid crystal.

TABLE II. The plateau value of the correlation function $C(X / 2)$, as a function of the surface coverage $c$ for each simulated system.

\begin{tabular}{ccccccc}
\hline \hline$c$ & Reference & $S=0.53$ & $Y=25.0$ & $m=3$ & $\delta_{x}=0$ & $\delta_{x}=X / 2 N$ \\
\hline 0.00 & 1.00 & 1.00 & & & & \\
0.15 & 0.93 & 1.00 & 0.92 & 0.91 & & \\
0.20 & 0.77 & 0.97 & 0.86 & 0.92 & 1.00 & 1.00 \\
0.25 & 0.34 & 0.65 & 0.52 & 0.84 & 1.00 & 1.00 \\
0.30 & 0.48 & 0.23 & 0.34 & 0.50 & 1.00 & 0.72 \\
0.35 & 0.32 & 0.48 & 0.35 & 0.15 & 1.00 & -0.07 \\
0.40 & 0.55 & 0.50 & 0.28 & 0.26 & 1.00 & -0.07 \\
\hline \hline
\end{tabular}


TABLE III. The correlation length $L_{C}$, defined by $C\left(L_{C}\right)=[1+C(X / 2)] / 2$, as a function of $c$ for each simulated system. A hyphen indicates that $L_{C}$ is undefined since $C(X / 2)=1$.

\begin{tabular}{ccccccc}
\hline \hline$c$ & Reference & $S=0.53$ & $Y=25.0$ & $m=3$ & $\delta_{x}=0$ & $\delta_{x}=X / 2 N$ \\
\hline 0.00 & - & - & & & & \\
0.15 & 16.9 & - & 13.8 & 17.9 & & - \\
0.20 & 29.0 & 34.4 & 14.6 & 17.3 & - & - \\
0.25 & 18.0 & 27.0 & 21.5 & 18.2 & - & 28.2 \\
0.30 & 20.9 & 29.7 & 16.2 & 21.6 & - & 40.3 \\
0.35 & 27.2 & 18.4 & 18.9 & 20.1 & - & 44.3 \\
0.40 & 20.1 & 22.1 & 20.7 & 24.4 & - & \\
\hline \hline
\end{tabular}

\section{Optical output}

The quantifications obtained by analyzing the correlation functions of the tensor order parameter are instructive but cannot be readily compared to the optical measurements made in actual experiments. Experimentally, the transversaloptical output of the system (rather than the lateral-optical output) is measured and correlated with the amount of ligand adsorbed at the walls. ${ }^{2,3}$ For our model sensor, we can compute the transversal-optical output by modifying Eq. (19) in order to allow for the addition of phase shifts of multiple, adjacent cells. We obtain

$$
I=I_{0} \sin ^{2}(2 \alpha) \sin ^{2}\left(\sum_{j} \epsilon_{j} / 2\right) .
$$

The former equation establishes that the transversal-optical output is governed by two factors: First, the total phase shift,

$$
\sum_{j} \epsilon_{j}=\sum_{j} \frac{2 \pi d}{\lambda}\left[n_{\mathrm{eff}}\left(x, y_{j}\right)-n_{o}\right]
$$

depends on the local director at each grid cell through the local index $n_{\text {eff }}(\gamma)$; therefore, this factor is sensitive to inhomogeneities of the uniformly planar texture. The second factor, $\sin ^{2}(2 \alpha)$, depends on the angle $\alpha$ between the axis of the polarizer and the $x$ axis. Hence, this factor modulates the optical output of the system as a whole; the maximum output for any given configuration of the director field is obtained for $\alpha=\pi / 4$, while zero output is obtained when $\alpha=0$.

Figure 6 shows the transversal-optical output (with $\alpha$ $=\pi / 4)$ as a function of the particle concentration for (a) random-adsorption systems and (b) periodic-adsorption patterns. For all systems, the optical output decreases monotonically with increasing concentration. This is consistent with the progressive destruction of the uniform planar texture, because the phase retardation is maximal when the angle $\gamma$ $=\pi / 2$ (i.e., the director points along the $x$ axis). In Fig. 6 we also present fits to the data for each system, using the empirical form

$$
I / I_{0}=a\left[1+\tanh \left(-\frac{c-c_{i}}{w}\right)\right]+b .
$$

In this equation, $a$ is the maximum normalized intensity and $b$ is a base line. The parameter $c_{i}$ is the inflection point, while $w$ measures the width of the transition region where the intensity goes from its maximum to its base line level. Table IV presents the values of these parameters for each of the simulated systems. While the position of the inflection point $c_{i}$ does not change very much, the width $w$ can be reduced by as much as an order of magnitude from its reference value: the in-phase periodic system has the sharpest transition of the optical output, with a width $w$ that is only 0.11 times the reference value. In fact, the only case where $w$ is greater than in the reference is for the system with $m=3$ and even then the change is only $20 \%$.

Unfortunately, we cannot compare directly these results with those from experiments because, in our model, the director is confined to the $x y$ plane and the optical output is identically zero when the polars' orientation is set to $\alpha=0$. In the experimental system, when the texture becomes nonuni-
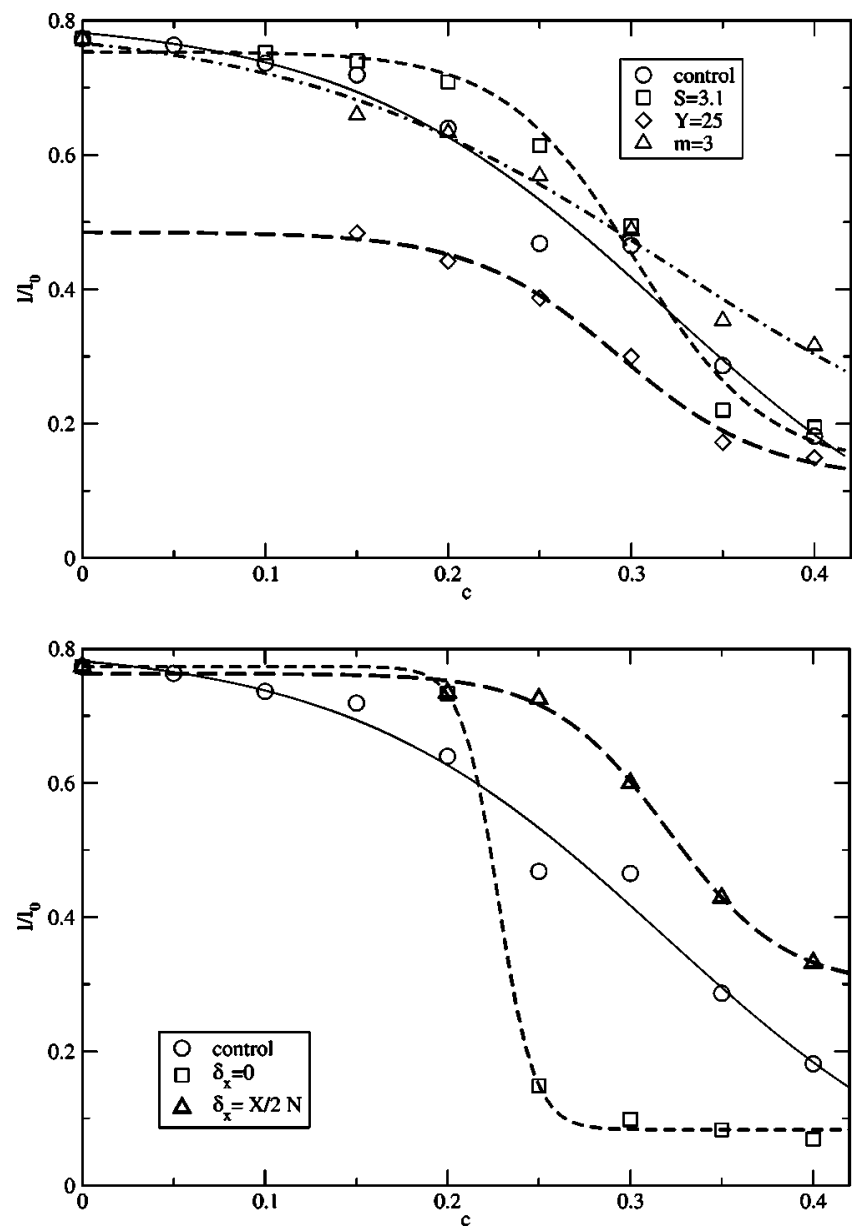

FIG. 6. Transversal-optical output (at $\alpha=0$ ), as a function of the surface coverage $c$, for (a) the random-adsorption systems and (b) the periodic adsorption systems. For comparison, the result for the reference system is also plotted in (b). 
TABLE IV. Parameters of the fit to Eq. (26), for the systems described in Table I.

\begin{tabular}{ccccccc}
\hline \hline & Reference & $S=0.53$ & $Y=25.0$ & $m=3$ & $\delta_{x}=0$ & $\delta_{x}=X / 2 N$ \\
\hline$a$ & 0.43 & 0.31 & 0.18 & 0.36 & 0.34 & 0.23 \\
$b$ & -0.06 & 0.14 & 0.12 & 0.07 & 0.08 & 0.30 \\
$c_{i}$ & 0.32 & 0.30 & 0.29 & 0.32 & 0.23 & 0.32 \\
$w$ & 0.18 & 0.072 & 0.082 & 0.21 & 0.020 & 0.063 \\
\hline \hline
\end{tabular}

form, the director is free to rotate out of the $x y$ plane. This explains why an increase in the luminance is observed at fixed orientation $\alpha=0$. In this sense, the lateral-optical output of the 2D sensor is more akin to the transversal output of the experimental 3D sensor: At zero surface coverage the uniform orientation results in a dark field; then, as more and more particles are added, the disorganized field starts to transmit light as a larger fraction of the local directors move away from the polarizer axis. As a result, the lateral-optical output at $\alpha=0$ increases with the particle concentration (see Fig. 7).

If we analyze the lateral-optical output as a function of $\alpha$ (the orientation of the polarizers with respect to the $x$ axis), we observe a sinusoidal modulation. For each system, we find that it is possible to fit the deviation with respect to the average intensity $(\bar{I})$ with the expression

$$
\frac{I(\alpha)-\bar{I}}{\bar{I}}=a_{0} \sin [4(\alpha+\phi)] .
$$

In this equation, $a_{0}$ is the amplitude of the modulation and $\phi$ is a constant phase.

Figure 8 shows the amplitude $a_{0}$ for the simulations in (a) the random-adsorption group and (b) the periodicadsorption group. In analogy with experiments, ${ }^{2,3}$ we observe a gradual decrease in the amplitude of modulation, in spite of the fact that the system remains at every case in the nematic phase. If we extrapolate the decrease of $a_{0}$ in order to find the coverage $c_{o}$ that would yield zero modulation, we find that $c_{o}$ is always larger than or equal to $c_{c}$, the critical coverage obtained from the correlation function.
Figure 8(b) shows that the in-phase system modulates light with the maximum amplitude for all surface coverages considered, as one expects for a uniform texture. In contrast, the out-of-phase system displays a decrease in the amplitude of modulation, but not as pronounced as that in the randomadsorption group.

\section{CONCLUSIONS}

First, we would like to refer to the investigations on the instability of uniform states of the order parameter against the perturbation of a random field. For the randomadsorption systems, the liquid crystal in the sensor does not readily disorganize into domains upon the addition of a few adsorbed particles. Instead, the disorganization is more gradual. However, for periodic-adsorption systems the limit of stability of the uniform texture depends on the lag $\delta_{x}$ between patterns in opposite walls; the correlation function $C(x)$ at the central region of the out-of-phase system decays rapidly towards zero, while that of the in-phase system remains practically constant and equal to unity.

These observations have several implications. First, the surface coverage is not sufficient to predict the critical amount of particles needed to destroy the uniform organization in the sensor; additional characterization of the adsorption pattern is required. This implies that one may be able to gain information about the morphology of the aggregation pattern from the sensor output. Second, taking the results for $m=3$ as typical, fewer particles could be detected if they adsorb in an individual fashion rather than in clusters, because the critical concentration $c_{c}$ and the width $w$ would be smaller. Also, if we take a cluster of particles as an approxi-

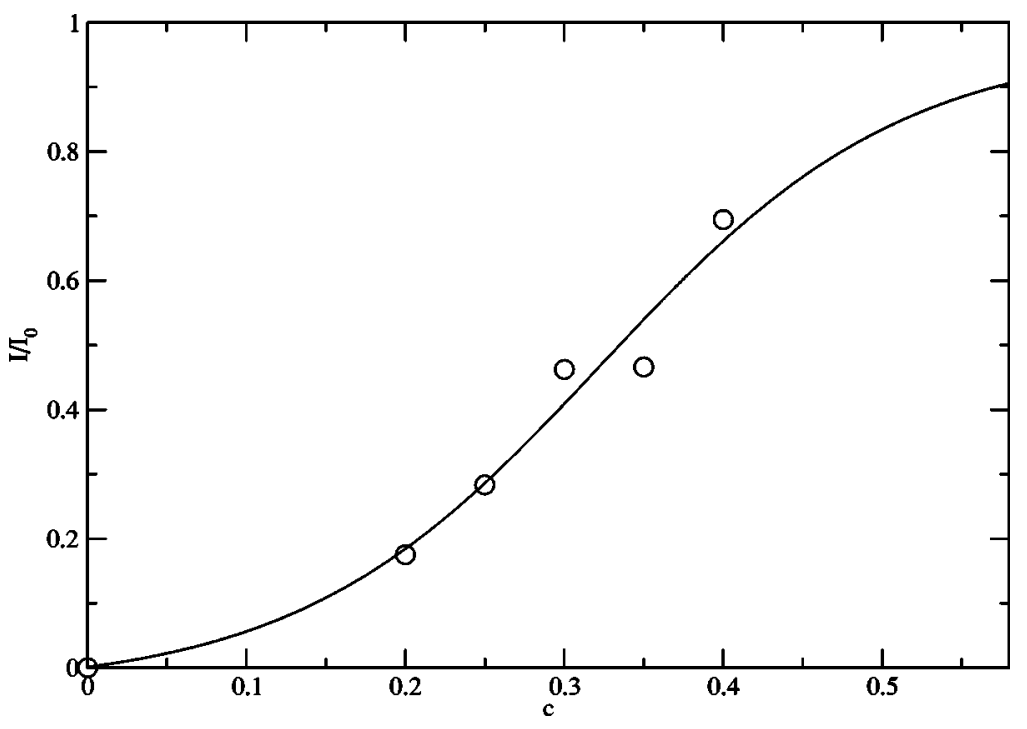

FIG. 7. Lateral-optical output for the reference system at $\alpha=0$ (the axis of the polarizer is parallel to the original uniform director), as a function of the surface coverage. At every value of $c$, the system is nematic, but the texture becomes increasingly nonuniform. 

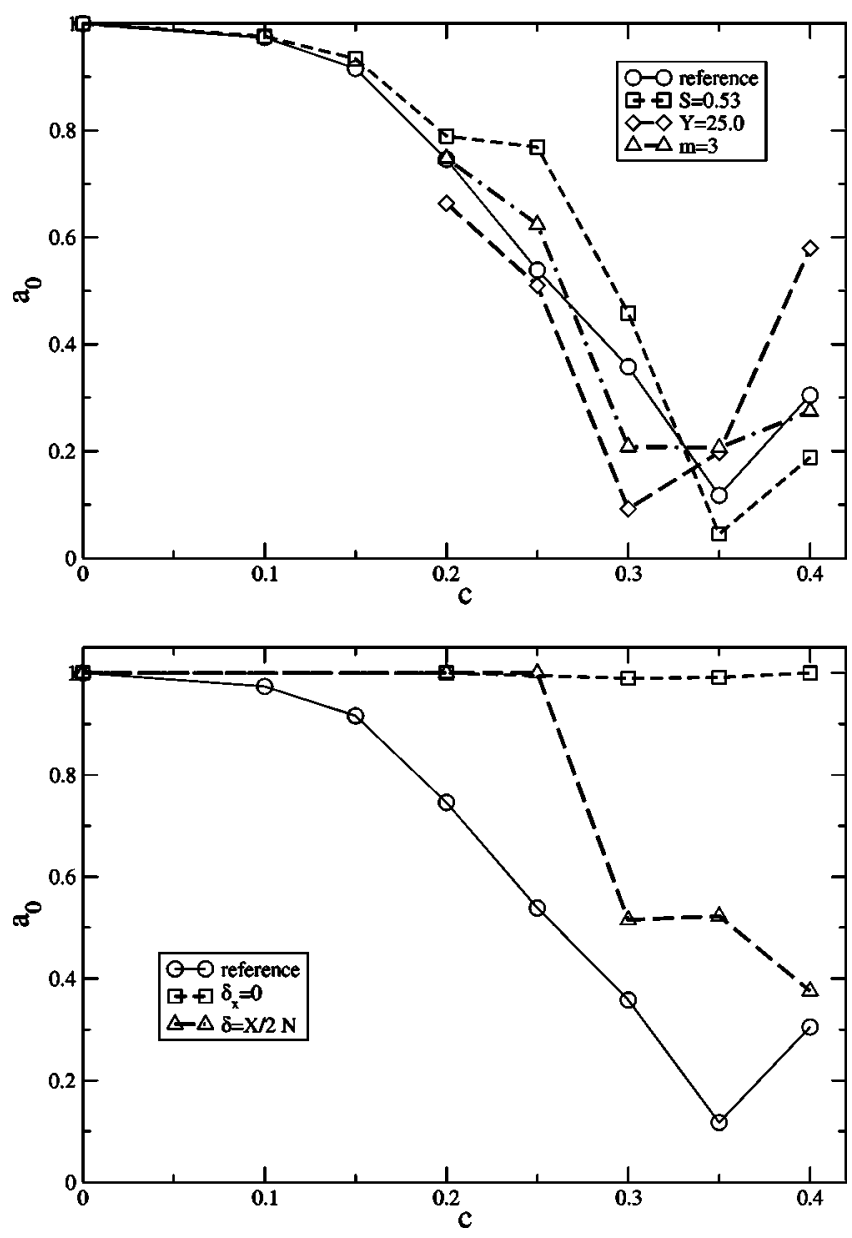

FIG. 8. Lateral-optical output of (a) the random-adsorption systems and (b) the periodic-adsorption systems, as a function of the surface coverage $c$. For comparison, the result for the reference system is plotted also in (b).

mation for a single elongated object, we can infer the effect of particle shape: we would expect elongated particles to result in higher values of $c_{c}$ and $w$, but the qualitative behavior would remain the same.

We expect that many of the trends observed in the quasi-2D sensor can be extrapolated to three-dimensional sensors. By decreasing the number of exploratory simulations that would be required, this would allow to select interesting regions of the parameter space for detailed analysis. Among those extrapolations, one can envision threedimensional analogs of the in-phase and out-of-phase systems, where 2D patterns in opposite walls can be shifted independently on the $x$ and $y$ directions; an interesting situation could be realized for systems that are in-phase along one direction but out-of-phase in the other.

In summary, we have presented results from an extensive set of simulations and investigated the effect that several variables that can be controlled in a liquid-crystal-based sensor have on the process of disorganization of the original uniform texture. The critical coverage $c_{c}$ can be used as a convenient description for the concentration needed in order for the liquid crystal to display a multidomain configuration.
However, the disorganization of the uniform state proceeds gradually as a function of the surface coverage, as evidenced by the smooth decline of the amplitude of the modulation of transmitted light by the entire system, and can be controlled by altering the adsorption pattern of the particles.

\section{ACKNOWLEDGMENTS}

This work was supported by the NSF-funded MRSEC on Nanostructured Interfaces at the University of Wisconsin, Madison. The authors are grateful to Dr. E. B. Kim for helpful discussions.

${ }^{1}$ V. K. Gupta, J. J. Skaife, T. B. Dubrovsky, and N. L. Abbott, Science 279, 2077 (1998).

${ }^{2}$ J. J. Skaife and N. L. Abbott, Langmuir 16, 3529 (2000).

${ }^{3}$ J. J. Skaife and N. L. Abbott, Langmuir 17, 5595 (2001).

${ }^{4}$ Y. Imry and S.-K. Ma, Phys. Rev. Lett. 35, 1399 (1975).

${ }^{5}$ T. Bellini, M. Buscaglia, C. Chiccoli, F. Mantegazza, P. Pasini, and C. Zannoni, Phys. Rev. Lett. 85, 1008 (2000).

${ }^{6}$ D. E. Feldman and V. M. Vinokur, Phys. Rev. Lett. 89, 227204 (2002).

${ }^{7}$ P. S. Clegg, C. Stock, R. J. Birgenau, C. W. Garland, A. Roshi, and G. S. Iannachione, Phys. Rev. E 67, 21703 (2003).

${ }^{8}$ S. Grollau, O. Guzmán, N. L. Abbott, and J. de Pablo, J. Chem. Phys. 122, 024703 (2005)

${ }^{9}$ B. Clare and N. L. Abbott, Langmuir (submitted).

${ }^{10}$ G. Goldbeck-Wood and A. H. Windle, Rheol. Acta 38, 548 (1999).

${ }^{11}$ A. Sonnet, A. Kilian, and S. Hess, Phys. Rev. E 52, 718 (1995).

${ }^{12}$ G. Rienacker, A. Kroger, and S. Hess, Physica A 315, 537 (2002).

${ }^{13}$ S. Grollau, E. B. Kim, O. Guzmán, N. L. Abbott, and J. J. de Pablo, J. Chem. Phys. 119, 2444 (2003).

${ }^{14}$ O. Guzmán, E. B. Kim, S. Grollau, N. Abbott, and J. J. de Pablo, Phys. Rev. Lett. 91, 235507 (2003).

${ }^{15}$ E. B. Kim, O. Guzmán, S. Grollau, N. L. Abbott, and J. J. de Pablo, J. Chem. Phys. 121, 1949 (2004).

${ }^{16} \mathrm{P}$. G. de Gennes and J. Prost, The Physics of Liquid Crystals (Clarendon, Oxford, 1993).

${ }^{17}$ P. M. Chaikin and T. C. Lubensky, Principles of Condensed Matter Physics (Cambridge University Press, Cambridge, UK, 1995).

${ }^{18}$ M. Zapotocky, P. M. Goldbart, and N. Goldenfeld, Phys. Rev. E 51, 1216 (1995).

${ }^{19}$ C. Denniston, E. Orlandini, and J. M. Yeomans, Phys. Rev. E 64, 21701 (2001).

${ }^{20}$ D. Andrienko, M. Tasinkevych, P. Patricio, M. P. Allen, and M. M. Telo da Gama, Phys. Rev. E 68, 51702 (2003).

${ }^{21}$ M. Tasinkevych, N. M. Silvestre, P. Patricio, and M. M. Telo Da Gama, Eur. Phys. J. E 9, 341 (2002).

${ }^{22}$ G. Toth, C. Denniston, and J. M. Yeomans, Phys. Rev. Lett. 88, 105504 (2002).

${ }^{23}$ M. Plischke and B. Bergersen, Equilibrium Statistical Physics, 2nd ed. (World Scientific, Singapore, 1994).

${ }^{24}$ D. P. Landau and K. Binder, A Guide to Monte Carlo Simulations in Statistical Physics (Cambridge University Press, Cambridge, 2000).

${ }^{25}$ A. N. Beris and B. J. Edwards, Thermodynamics of Flowing Systems: With Internal Microstructure (Oxford University Press, New York, 1994).

${ }^{26}$ B. J. Edwards and A. N. Beris, J. Rheol. 33, 1189 (1989).

${ }^{27}$ M. J. Stephen and J. P. Straley, Rev. Mod. Phys. 46, 617 (1974).

${ }^{28}$ J. Nehring and A. Saupe, J. Chem. Phys. 56, 5527 (1972).

${ }^{29}$ D. E. Feldman, Phys. Rev. Lett. 84, 4886 (2000).

${ }^{30}$ N. Schopohl and T. J. Sluckin, Phys. Rev. Lett. 59, 2582 (1987).

${ }^{31}$ L. A. Tecero Espinoza, K. R. Schumann, Y.-Y. Luk, B. A. Israel, and N. L. Abbott, Langmuir 20, 2375 (2004).

${ }^{32}$ T. M. Nicholson, Mol. Cryst. Liq. Cryst. 177, 163 (1989).

${ }^{33}$ V. K. Gupta and N. L. Abbott, Langmuir 15, 7213 (1999).

${ }^{34}$ For the calculation of the optical textures, we have used typical values for the refractive indices $n_{e}=1.7$ and $n_{o}=1.5$, and a short wavelength $(\lambda$ $=400 \mathrm{~nm}$ ) to compensate for the fact that the model sensor is smaller than the actual experimental devices. 